(C) 2011 IEEE. Personal use of this material is permitted. Permission from IEEE must be obtained for all other uses, in any current or future media, including reprinting/republishing this material for advertising or promotional purposes, creating new collective works, for resale or redistribution to servers or lists, or reuse of any copyrighted component of this work in other works. 


\title{
Measurement of Activity Produced by Low Energy Proton Beam in Metals Using off-line PET Imaging
}

\author{
P.M.G. Corzo, J. Cal-González, Student Member, IEEE, E. Picado, S. España Member, IEEE, J.L. Herraiz Member, \\ IEEE, E. Herranz Student Member, IEEE, E. Vicente Student Member, IEEE, J.M. Udías Member, IEEE, J.J. \\ Vaquero Senior Member, IEEE, A. Muñoz-Martín, L.M. Fraile Member, IEEE
}

\begin{abstract}
In this work, we investigate PET imaging with ${ }^{68} \mathbf{G a}$ and ${ }^{66} \mathrm{Ga}$ after proton irradiation on a natural zinc foil. The nuclides ${ }^{68} \mathrm{Ga}$ and ${ }^{66} \mathrm{Ga}$ are ideally suited for off line PET monitoring of proton radiotherapy due to their beta decay halflives of 67.71(9) minutes and 9.49(3) hours, respectively, and suitable $\beta$ end point energy. The purpose of this work is to explore the feasibility of PET monitoring in hadrontherapy treatments, and to study how the amount of activity and the positron range affect the $P E T$ image reconstruction. Profiting from the low energy reaction threshold for production via $(p, n)$ reactions, both ${ }^{68} \mathrm{Ga}$ and ${ }^{66} \mathrm{Ga}$ gallium isotopes have been produced by activation on a natural zinc target by a proton pencil beam. In this way, it is possible to create detailed patterns, such as the Derenzo inspired one employed here. The proton beam was produced by the $5 \mathrm{MV}$ tandetron accelerator at CMAM in Madrid. The energy of this beam (up to $10 \mathrm{MeV}$ ) is similar to the residual energy of the protons used for therapy at the distal edge of their path. The activated target was imaged in an ARGUS small animal PET/CT scanner and reconstructed with a fully 3D iterative algorithm, with and without positron range corrections.
\end{abstract}

\section{INTRODUCTION}

External beam radiotherapy using protons has been used extensively for more than forty years. Protons show an increasing energy deposition with the penetration distance, giving rise to the maximum of the energy loss -the Bragg peak- close to the end of the range of the protons. This

Manuscript received November 15, 2011. This work was supported in part by Comunidad de Madrid (ARTEMIS S2009/DPI 1802), Spanish Ministry of Science and Innovation (grants FPA2010 17142 and ENTEPRASE, PSE 3000002009 5), by European Regional Funds, by CDTI under the CENIT Programme (AMIT Project), UCM (grupos UCM, 910059) and by CPAN, CSPD 2007 00042@Ingenio2010.

P.M.G Corzo, J. Cal Gonzalez, E. Picado, E. Herranz, E. Vicente, J.M. Udías and L.M. Fraile are with the Grupo de Física Nuclear, Dpto. Física Atómica, Molecular y Nuclear, UCM, CEI Moncloa, Madrid, Spain, (telephone: +34913944484, e mail: jacobo@nuclear.fis.ucm.es, pablo@nuclear.fis.ucm.es, fraile@nuc2.fis.ucm.es)

E. Vicente is also with Instituto de Estructura de la Materia, Consejo Superior de Investigaciones Científicas (CSIC), Madrid, Spain.

S. España was with the Grupo de Física Nuclear, Universidad Complutense de Madrid, Spain. He is now with the Medical Image and Signal Processing Group, Dep. of Electronics and Information Systems, Ghent Univ. Hospital, Belgium

J.L. Herraiz was with the Grupo de Física Nuclear, Universidad Complutense de Madrid, Spain. He is now with Madrid MIT M+Visión consortium.

J.J. Vaquero is with the Departamento de Bioingeniería e Ingeniería Aeroespacial, Universidad Carlos III de Madrid, Spain.

A. Muñoz Martín is with the Centro de Microanálisis de Materiales, Universidad Autónoma de Madrid, Madrid, Spain. physical feature causes an advantage of proton treatment over photon or electron irradiation since the region of maximum energy deposition can be well positioned within the target for each beam direction. This opens up the possibility of achieving a highly conformal high dose region, created by a spread-out Bragg peak (SOBP), and thus the potential of covering extended tumour volumes with high accuracy and low collateral damage to healthy tissue [1].
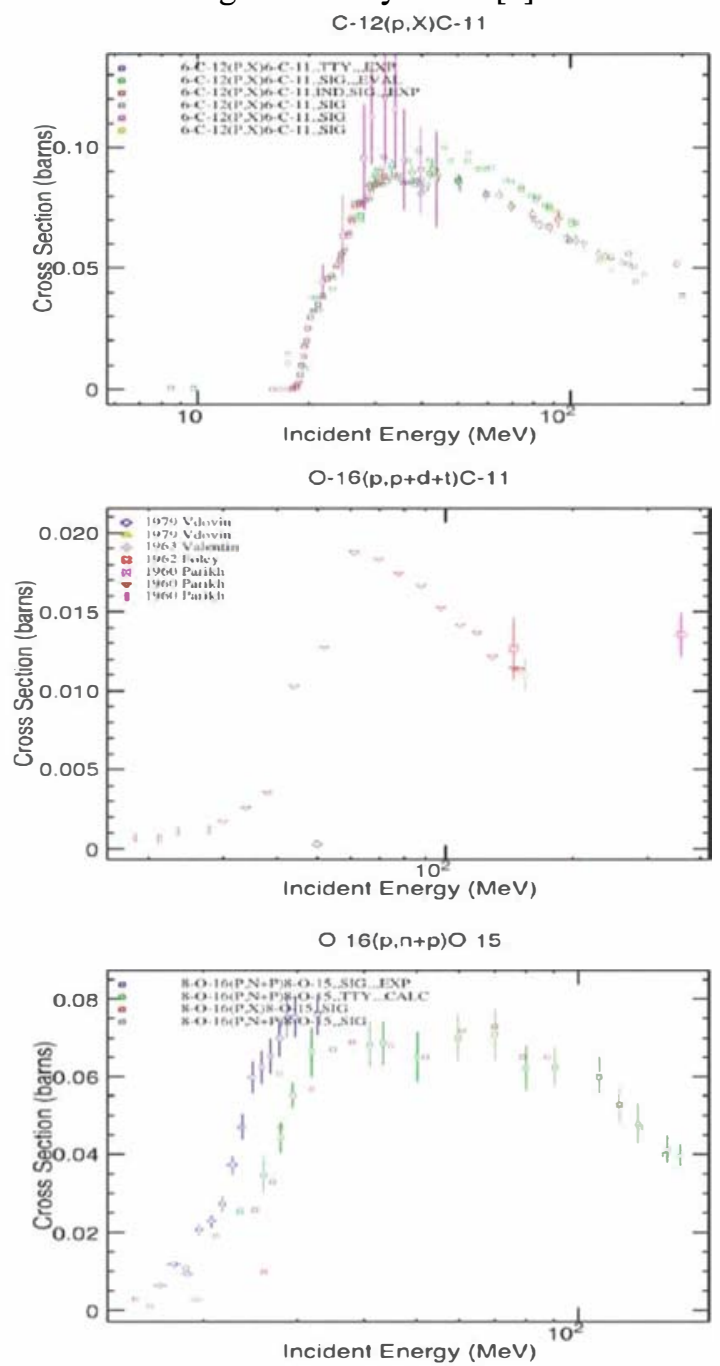

Fig. 1. Cross sections for production of ${ }^{11} \mathrm{C}$ and ${ }^{15} \mathrm{O}$ by protons impinging on stable carbon and oxygen isotopes $\left({ }^{12} \mathrm{C}\right.$ and $\left.{ }^{16} \mathrm{O}\right)[3]$.

The most promising method for in vivo and non-invasive monitoring of proton radiotherapy is positron emission 
tomography (PET) [2]. When suitable positron emitters, such as ${ }^{11} \mathrm{C}$ and ${ }^{15} \mathrm{O}$, are produced by nuclear interactions along the proton beam path, they can be imaged as a spatial imprint of dose deposition. The main complications of the method are the lack of activation in the few last millimeters of the penetration depth (see figure 1), which is due to the energy threshold of about 15 to $20 \mathrm{MeV}$ for most proton induced nuclear reactions, as well as the poor spatial correlation between the $\beta^{+}$ activity and the dose depth profiles. This hampers the extraction of appropriate information about the range and dose localization [1]. As seen in figure 1 the energy thresholds for the main $(p, p n)$ reaction channels leading to the production of ${ }^{11} \mathrm{C}$ and ${ }^{15} \mathrm{O}$ are $16.6 \mathrm{MeV}$ and $20.3 \mathrm{MeV}$ [3].

However, other so-called metal $\beta^{+}$isotopes suitable for PET imaging can be produced at lower energies than ${ }^{11} \mathrm{C}$ and ${ }^{15} \mathrm{O}$ via proton induced reactions on appropriate targets. If a given tumor-specific molecule is labeled with the target isotope for these reactions, the interesting $\beta^{+}$PET isotopes will be then produced in proton therapy by protons reaching the target volume with low energy. Some of these alternative PET nuclides are isotopes of $\mathrm{Ga}$ and $\mathrm{Cu}$ [4], [5]. In this experiment we would like to focus on the study of the gallium isotopes ${ }^{66} \mathrm{Ga}$ and ${ }^{68} \mathrm{Ga}$ (see Table I) with half-lives of $\mathrm{T}_{1 / 2} \quad$ 9.49(3) $\mathrm{h}$ and $\mathrm{T}_{1 / 2}$ 67.71(9) min respectively [6]. In a real patient irradiation at high energies, the total production of ${ }^{11} \mathrm{C}$ and ${ }^{15} \mathrm{O}$ isotopes would be much higher than what can be expected for the metal isotopes, but due to the reaction threshold it will occur at a different location. Moreover, since the half-life of these metal isotopes is larger than ${ }^{11} \mathrm{C}\left(\mathrm{T}_{1 / 2} \quad 20.334(24) \mathrm{min}\right)$ and ${ }^{15} \mathrm{O}\left(\mathrm{T}_{1 / 2} \quad 2.037(3) \mathrm{min}\right)$, the PET acquisition a few ${ }^{11} \mathrm{C}$ half-lives after proton irradiation will only retain the activity coming from metal isotopes.

TABLE I. MAIN PROPERTIES OF THE RADIONUCLIDES ${ }^{68} \mathrm{GA}$ AND ${ }^{66} \mathrm{GA}$, ALONG

\begin{tabular}{cccc}
\multicolumn{4}{c}{ WITH THE PET STANDARD ${ }^{18} \mathrm{~F}$. DATA TAKEN FROM [6]. } \\
\hline ISOTOPE & ${ }^{18} \mathrm{~F}$ & ${ }^{68} \mathrm{Ga}$ & ${ }^{66} \mathrm{Ga}$ \\
\hline $\mathrm{J}^{\pi}$ & $1^{+}$ & $1^{+}$ & $0^{+}$ \\
$\mathrm{T}_{1 / 2}(\mathrm{~min})$ & $109.77(5)$ & $67.71(9)$ & $569(2)$ \\
Stable daughter & ${ }^{18} \mathrm{O}$ & ${ }^{68} \mathrm{Zn}$ & ${ }^{66} \mathrm{Zn}$ \\
Annihilation branching & $194 \%$ & $178 \%$ & $112 \%$ \\
$\mathrm{Q}_{\beta}(\mathrm{keV})[$ branching] & $634[97 \%]$ & $822[1.2 \%]$ & $924[4 \%]$ \\
& & $1899[87.9 \%]$ & $4153[50 \%]$ \\
Mean $\mathrm{E}_{\beta}(\mathrm{keV})[$ branching] & $250[97 \%]$ & $353[1.2 \%]$ & $397[4 \%]$ \\
& & $836[87.9 \%]$ & $1905[50 \%]$ \\
Mean beta range $(\mathrm{mm})$ & 0.64 & 2.24 & 5.12 \\
\hline
\end{tabular}

In this experimental work, we use a low energy proton beam on a natural zinc target, as in Sattari et al [7]. We depict a Derenzo-inspired pattern on the target in order to evaluate the viability of producing phantoms for PET Imaging with Gallium isotopes.

\section{EXPERIMENT}

The Cockcroft-Walton $5 \mathrm{MV}$ tandetron accelerator at CMAM provides a proton beam of up to $10 \mathrm{MeV}$ [8]. As a compromise of sizable reaction cross-sections and reliable accelerator performance we used the CMAM proton beam at 9.0 $\mathrm{MeV}$ and $10 \mathrm{nA}$. The activation was performed in the standard multipurpose beam line at CMAM (see figure 2), which includes an experimental chamber with a 4-axes programmable goniometer with enough precision and speed in its axial and radial degrees of freedom for our purpose.

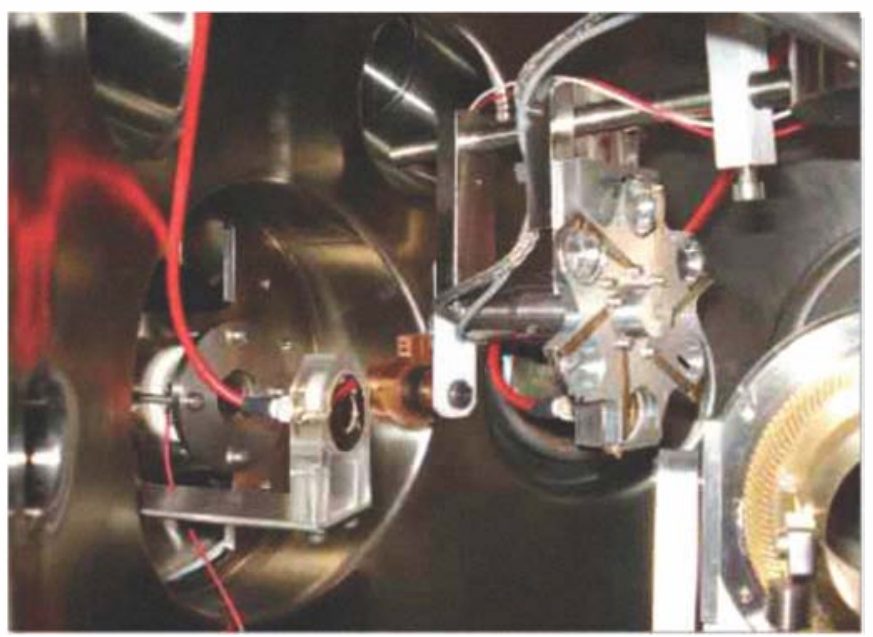

Fig. 2. The experimental chamber at the standard beam line at CMAM [8]

A high-purity $(99.99 \%)$ zinc target $(\rho=7.13 \mathrm{~g} / \mathrm{cm} 3)$ was used in this experiment, with natural abundances of $27.9 \%$ for ${ }^{66} \mathrm{Zn}$ and $18.8 \%$ for ${ }^{68} \mathrm{Zn}$. In a natural zinc target both gallium emitters of interest are produced with enough activity and adequate half-life to allow for an offline PET measurement. The different half-lives and Q-values of ${ }^{66} \mathrm{Ga}$ and ${ }^{68} \mathrm{Ga}$ allow exploring different activity ranges and positron range effects. Our aim was to activate a suitable target foil with a proton beam of this sort and activate a Derenzo-inspired pattern (figure 3). The irradiation time and intensity were adjusted in order to have the same activity per surface unit in each spot. The values are shown in Table II.

TABLE II. IRRADIATION TIME AND INTENSITY ADJUSTED TO HAVE THE SAME

\begin{tabular}{ccccc}
\multicolumn{5}{c}{ ACTIVITY PER SURFACE UNIT IN EACH SPOT } \\
\hline$\theta(\mathrm{deg})$ & $\mathrm{r}(\mathrm{mm})$ & $\begin{array}{c}\text { Size } \\
(\mathrm{mm})\end{array}$ & Time $(\mathrm{s})$ & $\mathrm{I}(\mathrm{nA})$ \\
\hline 108 & 5,13 & $3 \times 3$ & $2 \times 40$ & 6.3 \\
180 & $2,8,14$ & $2 \times 2$ & $3 \times 36$ & 3.1 \\
252 & $2,5,8,11,14$ & $1 \times 1$ & $5 \times 39$ & 0.78 \\
\hline
\end{tabular}

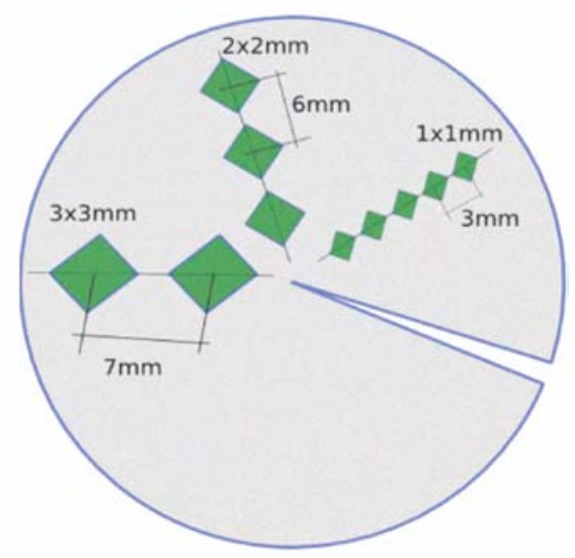

Fig. 3. Derenzo inspired pattern activated with the low energy proton beam at CMAM. 
An activimeter was used to measure the activity in the target after irradiation. The target with the Derenzo-inspired pattern was measured in the ARGUS small animal PET/CT scanner [9] located at the Laboratorio de Imagen Médica at the Hospital General Universitario Gregorio Marañón, and the acquisition was reconstructed with the $3 \mathrm{D}$ iterative reconstruction algorithm FIRST [10], with and without positron range correction [11]. After PET measurements, and in order to measure the activity of other isotopes produced (mainly ${ }^{67} \mathrm{Ga}$ and isotopes produced by other reactions channels) high-resolution gamma spectrometry with HPGe detector was performed, see figure 4 .

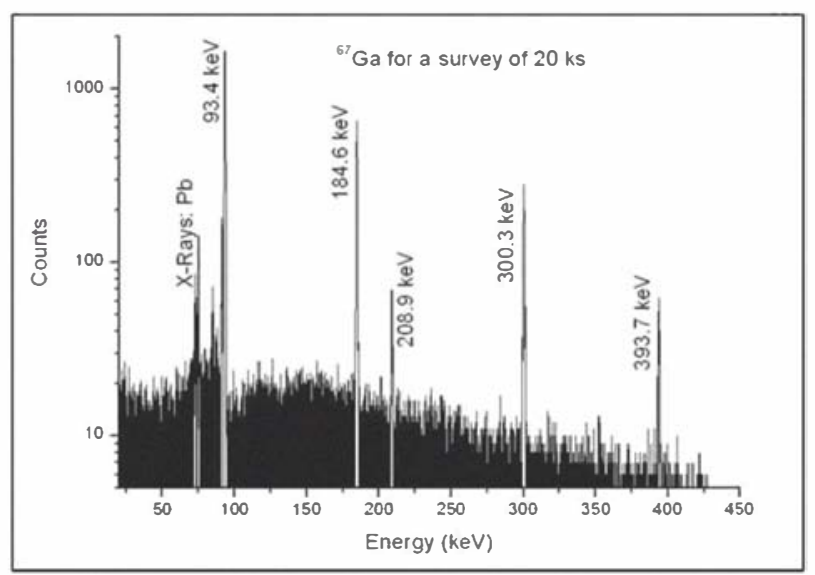

Fig. 4. Low energy region of the gamma spectrum obtained 8 days after irradiation with an HPGe detector. The full energy peaks corresponding to ${ }^{67} \mathrm{Ga}$ isotope are shown.

\section{COUNT RATE ESTIMATES FOR ${ }^{68} \mathrm{GA}$ AND ${ }^{66} \mathrm{GA}$}

For the count rate estimates we calculate the radionuclides produced per unit time in a thick target by a beam of protons of energy $\mathrm{E}$ as:

$$
\Gamma \equiv \phi Y(E)
$$

where $\Phi$ is the proton flux ( $\mathrm{s}^{1}$ ) for the selected beam current and $Y(E)$ is the thick target yield. Given the almost linear dependence of the cross section with penetration depth (see figure 5), which takes into account the behavior of the energy loss, we can estimate the amount of radionuclides produced per unit time using:

$$
\Gamma \equiv \phi f \rho_{A} r \hat{\sigma}
$$

where $\rho_{A}$ is the atomic density of natural $\mathrm{Zn}, f$ the fraction of the isotope of interest in the target material, $r$ is the penetration depth of the protons in the material for which the cross section is non-negligible, and $\hat{\sigma}$ is the cross section for protons in $\mathrm{Zn}$, as depicted in figure 6.

\section{RESULTS}

\section{A. Measured activity}

After 2.25 hours of the irradiation, we measured a total activity of $1.77 \mu \mathrm{Ci}$ for ${ }^{66,68} \mathrm{Ga}$ isotopes, which correspond to $65.5 \mathrm{kBq}$ of $511 \mathrm{keV}$ photons (see Table III). This activity differs only by $5.6 \%$ from the activity estimated from the reaction cross-sections and the irradiation times, obtained using the expression (2) above. This difference is smaller than the precision of the activimeter.

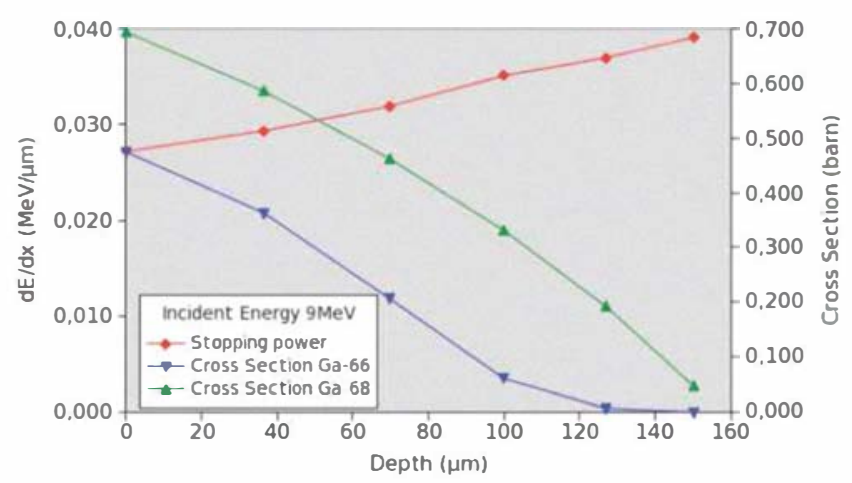

Fig. 5. Cross sections (barn) for ${ }^{68} \mathrm{Ga}$ and ${ }^{66} \mathrm{Ga}$ and energy loss as a function of depth in the $\mathrm{Zn}$ target. The data points are plotted every $1 \mathrm{MeV}$ starting at $9 \mathrm{MeV}$.

\begin{tabular}{|c|c|c|c|}
\hline$E(M / r V)$ & 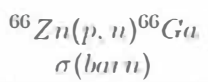 & $\begin{array}{c}{ }^{68} Z n(p, n)^{68} G i n \\
\sigma(l u n)\end{array}$ & range $(/ \mathrm{m} l)$ \\
\hline 11.0 & $0.6(08$ & $(0.838$ & 35.5 \\
\hline 10.0 & 0.5 .53 & (0.781 & 3() 2 \\
\hline 9.0 & 0.475 & ().694 & 253 \\
\hline 8.0 & 0.363 & 0.586 & 208 \\
\hline 7.0 & 0.206 & 0.462 & 166 \\
\hline $6 .()$ & ().06() & (). 331 & 129 \\
\hline 5.0 & ().0()7 & (). 193 & 77 \\
\hline 4.0 & $(0.00()$ & (). (146 & 53 \\
\hline
\end{tabular}

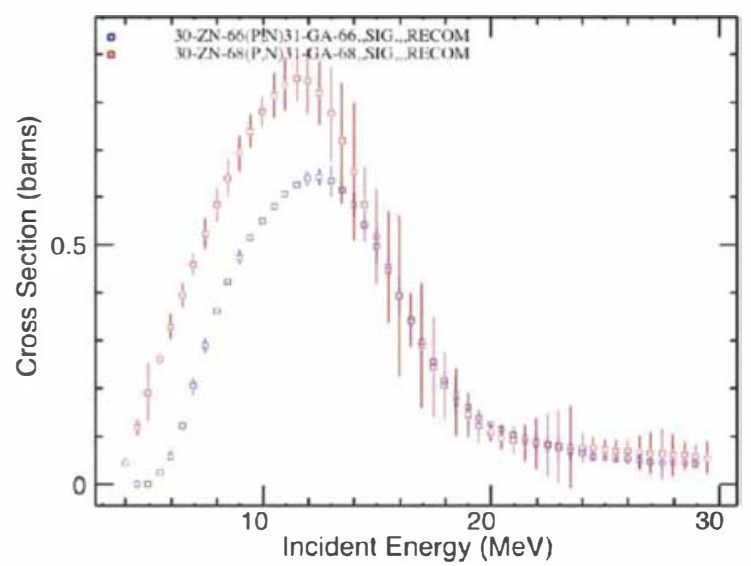

Fig. 6. Cross sections and projected range as a function of energy for the ${ }^{66} \mathrm{Zn}(\mathrm{p}, \mathrm{n}){ }^{66} \mathrm{Ga}$ and ${ }^{68} \mathrm{Zn}(\mathrm{p}, \mathrm{n}){ }^{68} \mathrm{Ga}$ reactions. PET acquisitions and image reconstructions

In order to study the activity evolution of the irradiated zinc foil, an acquisition with 27 frames (1200 seconds each) was acquired in the ARGUS small animal PET/CT scanner. Each frame was reconstructed using the FIRST procedure, with 80 updates of the image, without positron range correction. For each frame, the total number of counts was computed, obtaining the activity-time curve shown in figure 7 . This curve was fitted to the following expression:

$$
\operatorname{Counts}(t) \equiv A_{0} \operatorname{aexp}\left(-\frac{t}{\tau_{G a 68}}\right)+A_{0}(1-a) \exp \left(\begin{array}{c}
t \\
-\tau_{G a 66}
\end{array}\right)
$$

where $\mathrm{A}_{0}$ is the number of counts in the first frame, $\tau_{\mathrm{Ga} 68}$ and $\tau_{\mathrm{Ga66}}$ are the mean life for ${ }^{68} \mathrm{Ga}$ and ${ }^{66} \mathrm{Ga}$ respectively and $a$ is 
the fitting parameter which gives the ratio of ${ }^{68} \mathrm{Ga}$ in the first frame, 2.25 hours after irradiation. Using this fit

TABLE III. EXPECTED AND MEASURED ACTIVITIES FOR THE GA ISOTOPES
\begin{tabular}{|c|c|c|}
\hline \multicolumn{3}{|c|}{ Expected activity } \\
\hline Isotope & $\begin{array}{c}\text { Initial activity } \\
(\mathrm{kBq})\end{array}$ & $\begin{array}{c}\text { Activity after } 2.25 \\
\mathrm{~h}(\mathrm{kBq})\end{array}$ \\
\hline${ }^{66} \mathrm{Ga}$ & 18.4 & 15.2 \\
\hline${ }^{68} \mathrm{Ga}$ & 215 & 54.0 \\
\hline Measured activity after $\mathbf{2 . 2 5} \mathbf{~ h :} 65.5 \mathrm{kBq}$ total ${ }^{68} \mathrm{Ga}$ and ${ }^{66} \mathrm{Ga}$ \\
\hline
\end{tabular}

Using the proposed fit, the percentage of $80 \%$ of ${ }^{68} \mathrm{Ga}$ activity and $20 \%$ of ${ }^{66} \mathrm{Ga}$ activity 2.25 hours after irradiation is obtained, which compares well with the expected values of $78 \%$ and $22 \%$.

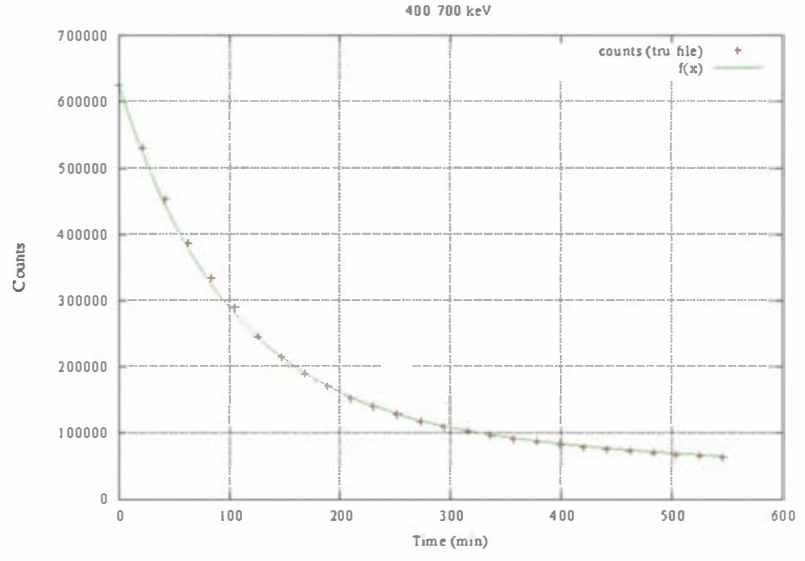

Fig. 7. Activity as a function of time obtained with the 27 frame acquisition fitted to the decay curve (3).

Figure 8 shows the PET reconstruction of the Derenzoinspired pattern irradiated in a zinc target, 2.25 hours after irradiation (first frame of the previous acquisition). The reconstruction was performed with the OSEM-3D code for the ARGUS scanner, without (left) and with positron range correction (right). The positron range correction was introduced into the reconstruction algorithm using the positron range profiles obtained from Monte Carlo simulations as an additional blurring applied to the object. This blurring can be adapted to the properties of the object in which the positrons are annihilated. The properties of the object were obtained from a CT image, using a simple segmentation in three different materials: zinc, water and air (see [11]).

\section{CONCLUSIONS}

The proposed experiment has been tested as a valid method for producing phantoms for PET imaging with Gallium isotopes. Moreover, no other proton induced reaction channels, which were open during the irradiation, disturbed our purposes either due to the longer half-life or the absence of positron emission. We have checked that the production cross-sections for Gallium isotopes are in good agreement with the EXFOR [3] tabulated ones. The irradiation of natural zinc by protons, as employed in this work, has been proved as a powerful method of preparing high resolution, activity calibrated, gallium phantoms which can be employed to test positron range corrected reconstruction methods.

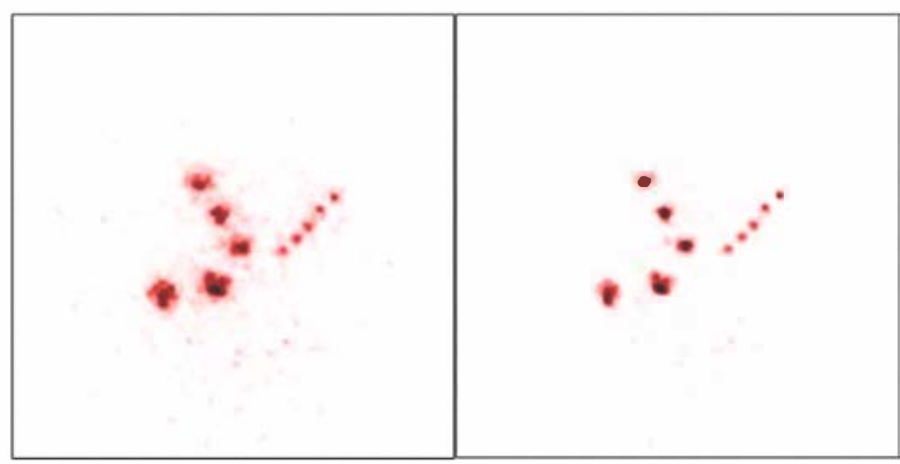

Fig. 8. PET reconstruction of the Derenzo inspired pattern irradiated in a zinc target, 2 hours after irradiation (first frame of the acquisition). Without range correction (left) and with range correction (right).

\section{ACKNOWLEDGMENTS}

We kindly acknowledge support from Comunidad de Madrid (ARTEMIS S2009/DPI-1802), Spanish Ministry of Science and Innovation (ENTEPRASE Grant, PSE-3000002009-5, FPA2010-17142) and PRECISION grant IPT-3000002010-3 and European regional funds and CPAN, Centro de Física de Partículas, Astropartículas y Nuclear (CSD-200700042@Ingenio2010-12). This study has been (partially) funded by CDTI under the CENIT Programme (AMIT Project).

\section{REFERENCES}

[1] D. Schardt, T. Elsässer and D. Schulz Ertner, Heavy ion tumor therapy: Physical and radiobiological benefits, Rev. Mod. Phys. 82, 383 425, 2010

[2] K. Parodi, W. Enghardt and T. Haberer, In beam PET measurements of $\beta^{+}$radioactivity induced by proton beams, Phys. Med. Biol. 47, 21 36, 2002

[3] EXFOR: Experimental Nuclear Reaction Data, http://www.nndc.bnl.gov/exfor/exfor00.htm

[4] C.J. Mathias, M.R. Lewis, D.E. Reichert, R. Laforest, T.L. Sharp, J.S. Lewis, Z. Yang, D.J. Waters, P.W. Snyder, P.S. Low, M.J. Welch and M.A. Green, Preparation of ${ }^{66} \mathrm{Ga}$ and ${ }^{68} \mathrm{Ga}$ labeled $\mathrm{Ga}(\mathrm{III})$ deferoxamine folate as potential folate receptor targeted PET radiopharmaceuticals, Nuclear Medicine and Biology 30, 725 731, 2003.

[5] Ö. Ugur, P.J. Kothari, R.D. Finn, P. Zanzonico, S. Ruan, I. Guenther, H.R. Maecke and S.M. Larson, Ga 66 labeled somatostatin analogue DOTA DPhe ${ }^{1} \mathrm{Tyr}^{3}$ octreotide as a potential agent for positron emission tomography imaging and receptor mediated internal radiotherapy of somatostatin receptor positive tumors, Nuclear Medicine and Biology 29, 147 157, 2002.

[6] Nuclear structure and decay data, http://www.nndc.bnl.gov/nudat2

[7] Sattari, A.; Shadanpoor, N.; Aslani,G., et al. Production of Ga 66 from natural zinc. Alasbimn Journal 8(31): January 2006. Article $\mathrm{N}^{\circ} \mathrm{AJ} 315$

[8] Centro de Microanálisis de Materiales, http://www.cmam.uam.es/.

[9] W. Yuchuan, J. Seidel, B.M.W. Tsui, J.J Vaquero, M.G. Pomper, "Performance Evaluation of the GE Helthcare eXplore VISTA Dual Ring Small Animall PET Scanner", J. Nucl. Med. Vol. 47 No.11 1891 1900.

[10] J. L. Herraiz, S. España, J. J. Vaquero, M. Desco, and J. M. Udías, FIRST: Fast Iterative Reconstruction Software for (PET) tomography, IEEE Trans. Nucl. Sci., 2003, vol. 50, pp. 398404.

[11] J. Cal González, J.L. Herraiz, S. España, E. Vicente, E. Herranz, M. Desco, J.J. Vaquero and J.M. Udías, Study of CT based positron range correction in high resolution 3D PET Imaging, Nucl. Instrum. Methods Phys. Res. A 172 175, 2011 